\title{
Constant-Frequency Control of Quasi-Resonant Converters
}

\author{
Dragan Maksimović Member, IEEE, and Slobodan Ćuk, Member, IEEE
}

\begin{abstract}
An additional independent control needed to eliminate the undesirable variable switching frequency of quasi-resonant converters can be obtained if the output rectifier is replaced by an active switch. The concept is applicable to all classes of quasi-resonant converters. It is demonstrated that in addition to operation at constant switching frequency, selected classes of constant-frequency quasi-resonant (CF-QR) topologies feature extended range of accessible conversion ratios and load currents. A practical example of a constant-frequency multi-resonant (CF-MR) buck converter operating at $2 \mathrm{MHz}$ is described.
\end{abstract}

\section{INTRODUCTION}

$\mathrm{Q}$ UASI-RESONANT (QR) converter topologies are generated by addition of resonant elements to a PWM (squarewave) converter. Consider, for example, the buck PWM converter in Fig. 1(a). The buck converter, just as all other basic PWM topologies, has two switches-an active (transistor) switch $S$ and a rectifier (diode) switch $\hat{S}$. If a resonant inductor and a resonant capacitor are added as shown in Fig. 1(b), the modified topology becomes a member of the zero-current quasiresonant (ZC) class [1]. Note that the $s$-switch needs to be converted into a two-quadrant switch. A current-bidirectional switch is used in this particular example. Other positions of the resonant elements, or addition of more than two resonant elements result in other possible QR topologies, such as zero-voltage (ZV) [2], quasi-square-wave (ZC-QSW, ZV-QSW) [3], [4], or multi-resonant (ZC-MR, ZV-MR) [5].

In contrast to PWM topologies, where the switch waveforms are approximately rectangular, QR converters exhibit smooth, quasi-sinusoidal waveforms and switching transitions at zero voltage or at zero current. For example, in the ZC topology, the $S$-switch is turned oN and OFF at zero current, while the $\hat{S}$-switch is turned ON and OFF at zero voltage, so that the power losses associated with switching transitions are greatly reduced. As a result of the reduction in the frequency-dependent part of the total power loss, higher switching frequency can be utilized, and correspondingly smaller values and sizes of energy-storage components should lead to smaller and lighter power converters. This is the main motivation behind the introduction of various classes of $\mathrm{QR}$ converters.

In comparison to $\mathrm{PWM}$ topologies, several unfavorable features of QR converters can be identified: voltage and current

Manuscript received September 20, 1989. This paper was presented at the 1989 High Frequency Power Conversion Conference, Naples FL, May 14-18. This work was conducted under the Power Electronics Program supported by grants from Boeing Electronics Company, GTE Communication Systems Corporation, Rockwell, Inc., and EG\&G Almond Instruments Inc.

D. Maksimović is with the Faculty of Electrical Engineering, University of Belgrade, Belgrade, Yugoslavia.

S. Cuk is with the Power Electronics Group, California Institute of Technology, Pasadena, CA 91125.

IEEE Log Number 9040465

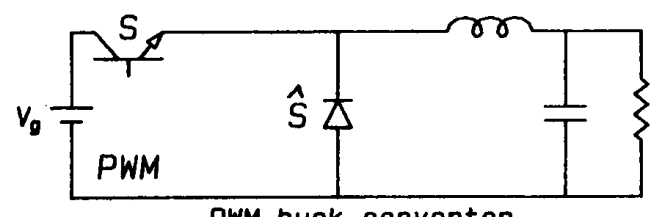

PWM buck converter

(a)

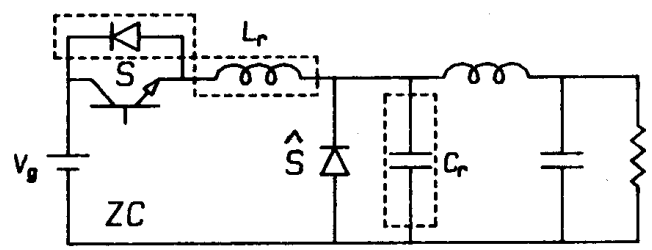

Zero-Current Quasi-Resonant buck

(b)

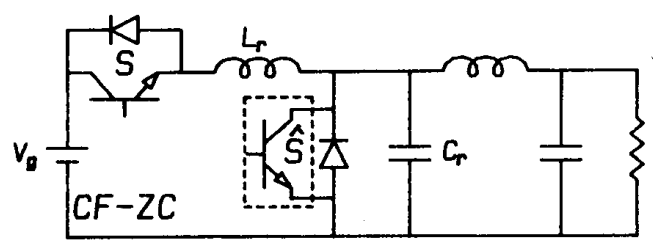

Constant-Frequency ZC buck

(c)

Fig. 1. Modification of PWM buck (a) yields quasi-resonant buck (b). Addition of controllable rectifier results in constant-frequency quasi-resonant buck topology (c). In each step, added elements are highlighted.

stresses on switching devices are higher; conduction losses are increased; load range and/or range of attainable conversion ratios are limited. Yet another disadvantage is that the switching frequency has to be varied in order to control the output voltage against variations in input voltage and load. The variable-frequency control is undesirable because optimal utilization of magnetic components is not possible. Furthermore, it is more difficult to handle the generated noise (conducted and radiated). Finally, it is not unusual that operation at constant frequency is imposed by system requirements.

In the ZC buck converter of Fig. 1(b), for example, the instant at which the $S$-switch is turned on can be determined by an external control signal, just as in the original PWM parent converter. However, the turn-off transition (at zero current) is confined to an interval determined by zero-crossing of the resonant current waveform. As a result, one degree of freedom is lost and only a variable-frequency control is applicable. The same conclusion holds true for all other quasi-resonant topologies. 
Several methods for constant-frequency control of resonant converters have been proposed. In all cases, an additional independent control is introduced in order to enable the operation at constant switching frequency. Thus, in [6], an additional switch is used to vary the apparent value of a resonant element. Conceptually similar is the method where the nonlinear characteristic of the magnetic material is used to control the value of a resonant inductor. Another possibility is to use full-bridge resonant topologies where a variety of control strategies can be devised for the four active (controllable) switches. Examples of constant-frequency, full-bridge resonant converters are discussed in [7] and [8].

In order to introduce the approach presented in this paper, suppose that the ZC buck converter of Fig. 1(b) is further modified as shown in Fig. 1(c). The $\hat{S}$-switch is converted into a controllable rectifier so that its turn-off transition is no longer subject to the circuit waveforms, i.e., the instant at which the $\hat{S}$-switch is turned ofF is determined by some external control. Hence, in the constant-frequency zero-current (CF-ZC) topology, two switching transitions are externally controllable-the $S$-switch turn-on and the $\hat{S}$-switch turn-off. The conversion ratio can be controlled by varying the length of the interval between the two controllable transitions, while the switching frequency is kept constant.

It is interesting that in [9], a controllable rectifier was used to achieve the constant-frequency control of converters that can be classified as members of the ZV-QSW class. In Section II, constant-frequency quasi-resonant converters are introduced in more general terms-the controllable rectifier can be implemented in any QR topology and obtained benefits are not restricted to the possibility of the constant-frequency control. In Sections III through V, operating modes, idealized waveforms, steady-state characteristics and design considerations are discussed for the three most interesting CF-QR classes-CF-ZV, CF-ZV-QSW, and CF-ZV-MR. A practical converter ( $2 \mathrm{MHz}$ $25 \mathrm{~W}$ CF-ZV-MR buck) is presented in Section VI together with experimental results.

\section{Quasi-Resonant Converters With a CONTROLlable RECTIFIER}

Quasi-resonant converters are derived by adding two or more resonant elements to a parent PWM converter. Basic PWM topologies have two switches-an active (controllable) switch implemented as a transistor (the $S$-switch), and a rectifier implemented as a diode (the $\hat{S}$-switch). Depending on the position of resonant elements with respect to the switches, various classes of QR converters can be identified. A complete account of possible $\mathrm{QR}$ topologies is given in [13]. In conventional $\mathrm{QR}$ topologies, the $S$-switch can be current bidirectional,

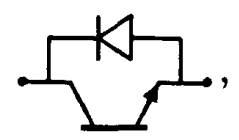

or voltage bidirectional,

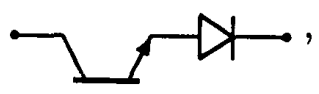

while the $\hat{S}$-switch is a diode. It is well known that substantially different operating modes (half-wave, full-wave) correspond to the different realizations of the active switch. It is not surprising that a number of novel operating modes can be found if both switches in a QR topology are controllable. Most importantly, the controllable rectifier provides the additional independent control necessary for operation at constant switching frequency. The term constant-frequency quasi-resonant (CF-QR) is used to denote the family of $Q R$ topologies with a controllable rectifier.

In a CF-QR topology, the two controllable switches can be current bidirectional or voltage bidirectional. In general, there are three distinct switch realizations:

CC: current-bidirectional \& current-bidirectional;

CV: current-bidirectional \& voltage-bidirectional;

VV: voltage-bidirectional \& voltage-bidirectional.

A separate study of possible operating modes is necessary for each of the three realizations. In this paper, we consider the realization with two current-bidirectional switches, which is of most practical interest for two reasons: voltage-bidirectional switches are prone to higher conduction losses because of the additional diode in series with the transistor; furthermore, the zero-voltage switching (which is more favorable than the zerocurrent switching) of a voltage-bidirectional switch is not possible in a practical converter because the diode in series with the transistor prevents resonant discharge of the transistor's parasitic capacitance.

The CF-QR classes that are particularly interesting for highfrequency applications-zero voltage (CF-ZV), zero-voltage quasi-square-wave (CF-ZV-QSW) and zero-voltage multi-resonant (CF-ZV-MR) - are studied in Sections III through V. The terminology and notation used in the subsequent sections are summarized as follows.

- An operating state of a converter is associated with position of the two switches. For a given QR converter topology, there can be up to four operating states, denoted as $(0-0),(1-0),(0-1)$, and (1-1). In each state, the position of the $S$-switch and the position of the $\hat{S}$-switch are indicated. For example, if the $S$-switch is on and the $\hat{S}$-switch is off, the converter is in the (1-0)-state, and so on.

- An operating mode is a periodic sequence of operating states. Of particular interest are the modes of CF-QR converters in which all switching transitions are at zero voltage or at zero current, so that switching losses are minimized, and in which two transitions are controllable, so that the constant-frequency control is applicable. The interval between the two controllable transitions (relative to the switching period) is defined as the control duty ratio $D_{c}$.

- In the load-to-output plane, the dc conversion ratio of a converter is shown against the load current. An operating region is a part of the load-to-output plane where operation in a given mode is possible. Take, as an example, the class of conventional $\mathrm{ZV}$ converters. It is well known that the zero-voltage switching is possible only if the converter's output current exceeds a certain minimum value [2]. Thus, the operating region of a $\mathrm{ZV}$ converter in the half-wave mode or in the full-wave mode cannot include the zeroload axis. Similar restrictions can be found for operating regions of other $\mathrm{QR}$ topologies. For the design of QR converters, boundaries of the operating region are of particular importance because one of the basic design considerations is to fit the required range of load currents and conversion ratios into the available operating region. 
TABLE I

NOTATION FOR NORMALIZED QUaNTITIES USED IN the Analysis of CF-QR Converters

\begin{tabular}{lc}
\hline \multicolumn{1}{c}{ Normalized Quantity } & Notation \\
\hline$S$-switch voltage, current & $v_{t}, i_{t}$ \\
$\hat{S}$-switch voltage, cutrent & $v_{d}, i_{d}$ \\
Load current & $\delta$ \\
Time & $\theta$ \\
Switching period & $\theta_{p}$ \\
Switching frequency & $f$ \\
\hline
\end{tabular}

- The resonant inductor, $L_{r}$, and the resonant capacitor, $C_{r}$, form a resonant network with

$$
\begin{aligned}
R_{o} & \equiv \sqrt{L_{r} / C_{r}}, \\
f_{r} & =\omega_{r} / 2 \pi \equiv 1 / 2 \pi \sqrt{L_{r} C_{r} .}
\end{aligned}
$$

- Voltages are normalized to the input voltage $V_{g}$, currents to $V_{g} / R_{o}$, frequency to the resonant frequency $f_{r}$ and time to $1 / \omega_{r}$. The notation for normalized quantities is summarized in Table I.

- Switch voltage and current stresses are relevant for comparison of various topologies. In the discussions of Sections III through $\mathrm{V}$, the switch voltage stress $V_{\text {stress }}$ is normalized to the voltage stress in the PWM parent. Similarly, the switch current stress $I_{\text {stress }}$ is normalized to the current stress in the PWM parent at the maximum output current.

\section{A. A Unifying Analysis Method}

Suppose that a $Q R$ converter has a dc conversion ratio $\mathfrak{N}$ ( $\mathfrak{N} \equiv V_{\text {our }} / V_{g}$ ), and that $M(D)$ is the conversion ratio of the PWM parent converter, where $D$ is the duty ratio of the $S$-switch. Recall that $M(D)=D$ for buck, $1 /(1-D)$ for boost, $-D /(1-D)$ for buck-boost and Ćuk converters, etc. The equivalent duty ratio $m$ of the QR converter is numerically equal to the duty ratio for which the PWM parent would have the same conversion ratio $\mathfrak{T}$, i.e., $m$ solves the equation $M(m)$ $=\mathfrak{N}$.

For any given QR class and a specified operating mode, $m$ (as a function of the normalized load $\delta$ and control variables) does not depend on the specific PWM parent topology [13]. In order to compute the DC conversion ratio for any particular QR topology, one should only substitute $m$ for duty ratio $D$ in the well-known function $M(D)$ of the PWM parent. This unifying method, originally devised in [10] for zero-current and zerovoltage QR classes, and applied to all QR classes in [13], renders unnecessary and redundant repeated analyses of numerous variations of $Q R$ topologies.

The results of Sections III through $\mathrm{V}$ are applicable to all CF-QR converters constructed from various PWM parents. In each section, a CF-QR buck topology (for which $\mathfrak{N}=M(m)$ $=m$ ) is included only to provide a reference for the computed waveforms and for the corresponding discussion.

\section{Constant-Frequency, Zero-Voltage CONVERTERS}

In a CF-ZV converter, the $S$-switch is switching at zero voltage, while the $\hat{S}$-switch is switching at zero-current, just as in conventional, frequency-controlled ZV converters. An example of the CF-ZV buck converter is shown in Fig. 2(a). There are two operating modes of interest:

$$
\begin{aligned}
& I: \cdots \rightarrow 0-0 \rightarrow 0-1 \rightarrow 1-1 \rightarrow 1-0 \rightarrow \cdots, \\
& I I: \cdots \rightarrow 1-1 \rightarrow 0-1 \rightarrow 1-1 \rightarrow 1-0 \rightarrow \cdots .
\end{aligned}
$$

The sequence of operating states in mode $I$ is identical to the one found for frequency-controlled $\mathrm{ZV}$ converters. If the current-bidirectional switches are used, mode $I$ is quite similar to the well-known half-wave mode. The only difference is that the transition from the (0-0)-state to the (0-1)-state occurs when the $\hat{S}$-switch is turned oN, before its voltage drops to zero, whereas in the half-wave mode the $\hat{S}$-switch (diode) starts to conduct when its voltage reaches zero. Mode $I$ is of less practical interest because the corresponding operating region is very restricted [12].

The periodic sequence of states in mode $I I$ is not encountered in the operating modes of frequency-controlled $\mathrm{ZV}$ converters. The following qualitative description of the operation in mode $I I$ is with reference to the pertinent waveforms shown in Fig. 2(b). Assume that initially the converter is in the (1-0)-state and that the $\hat{S}$-switch is turned on at the beginning of the switching cycle. The turn-on is at zero current. In the subsequent (1-1)-state, current $i_{d}$ descends linearly until the $s$-switch is turned OFF at $\theta=\theta_{1}$, at zero voltage, and the circuit enters the (0-1)-state in which $L_{r}$ and $C_{r}$ are allowed to resonate. The (0-1)-state terminates when the $S$-switch voltage reaches zero again so that its antiparallel diode starts to conduct at $\theta=\theta_{1}$. After the (1-1)-state is re-entered, the transistor in the $\hat{S}$-switch needs to be turned ofF during the interval when current $i_{d}$ is positive. Thus, when $i_{d}$ reaches zero at $\theta=\theta_{3}$, the $\hat{S}$-switch turns OFF, and the final (1-0)-state is entered. The transistor in the $S$-switch has to be turned on before current $i_{t}$ becomes positive. The switching cycle is completed when the $\hat{S}$-switch is turned on again at $\theta=\theta_{4}$.

Waveforms $p_{t}$ and $p_{d}$ denote control signals for the transitors in $S$ and $\hat{S}$, respectively. In the hatched areas, the state of the corresponding transistor is irrelevant for the operation of the circuit because the antiparallel diode is conducting.

Control duty ratio $D_{c}$ is defined as the interval between the two controllable transitions-the $\hat{S}$-switch turn-on and the $S$-switch turn-off-relative to the switching period $\theta_{p}$,

$$
D_{c} \equiv \theta_{1} / \theta_{p}
$$

dc analysis of mode $I I$ yields an expression for the equivalent duty ratio $m$ as a function of frequency $f$, load $\delta$ and control $D_{c}$.

$$
m=1-\frac{f}{\pi}\left[\pi-\arctan \left(\delta+D_{c} \theta_{p}\right)+\delta+D_{c} \theta_{p}\right] .
$$

The operating region for mode $I I$ is shown in Fig. 2(c) with normalized frequency $f$ as a varying parameter. For lower $f$, the operating region expands in both $\delta$ and $m$ directions. In contrast to frequency-controlled $\mathrm{ZV}$ converters, operation at zero load is possible. However, the range of attainable conversion ratios is more restricted. At zero load, the equivalent duty ratio cannot be greater than $1-f$. Suppose that the required equivalent duty ratio $m^{*}$ is determined from design specifications. Then, parameter $f$ has to satisfy

$$
f<1-m^{*} .
$$

The selection of $f$ affects the trade-off between the $S$-switch voltage stress and the switch current stress. Thus, if $f \rightarrow 1-$ 


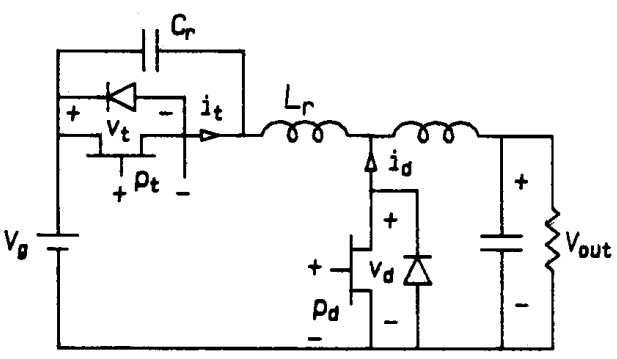

(a)

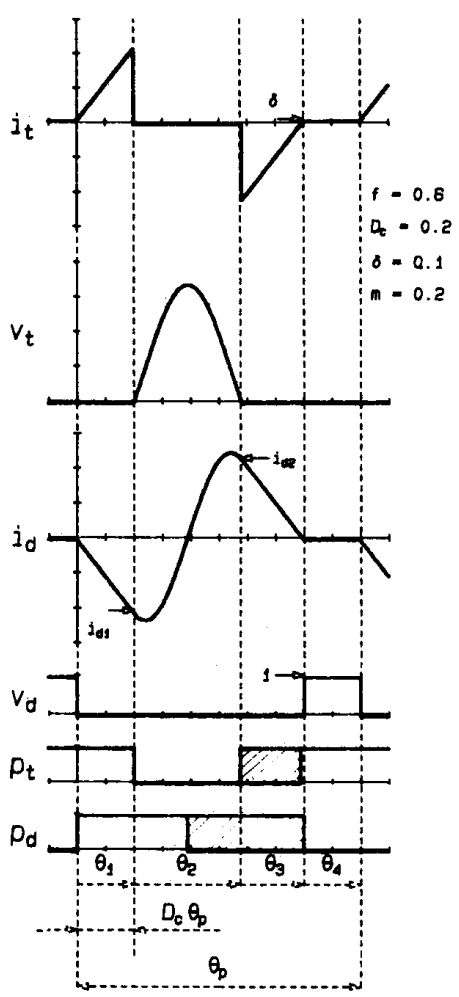

(b)

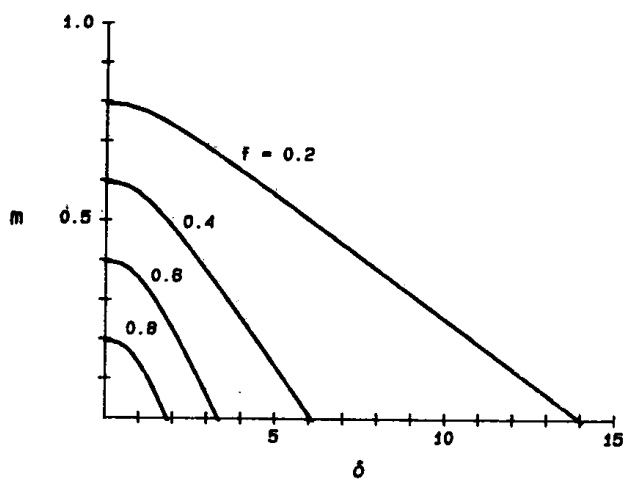

(c)

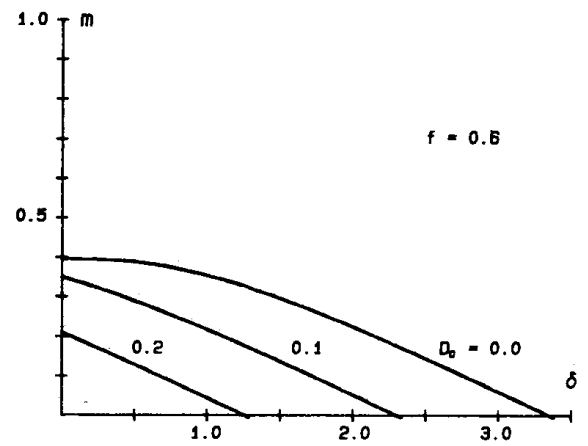

(d)

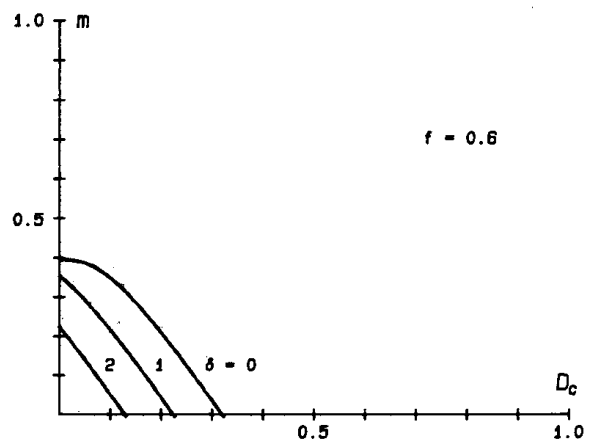

(e)

Fig. 2. (a) CF-ZV buck converter; (b) typical waveforms in mode $I I$; (c) operating region for mode $I I$, with normalized frequency $f$ as varying parameter; (d) load-to-output dc characteristics; (e) control-to-output dc characteristics.

$m^{*}$, the $S$-switch voltage stress tends to its minimum value ( $V_{\text {stress }} \rightarrow 2$ ), but the current stress becomes infinitely large. On the other hand, if $f \rightarrow 0$, the current stress tends to 1 (to the same value as in the PWM parent), but the voltage stress blows up. Within these limits, parameter $f$ can be selected to adjust the values of voltage and current stresses. Regardless of the choice for $f$, the $\hat{S}$-switch voltage stress is ideally equal to the switch voltage stress in the PWM parent.

As an example, for $m^{*}=0.6$, parameter $f$ is computed so that the $S$-switch stress-product, $V_{\text {stress }} \times I_{\text {stress }}$, is minimized. The result is

$$
\begin{aligned}
f & =0.32, \\
V_{\text {stress }} & =5.2, \\
I_{\text {stress }} & =2.2 .
\end{aligned}
$$

A conventional, frequency-controlled $\mathrm{ZV}$ converter would have the current stress equal to one. However, the CF-ZV converter has an unlimited load range (no load to full load), while the load range of the conventional $\mathrm{ZV}$ converter is numerically equal to the voltage stress minus one.

DC characteristics in the normalized load-to-output and control-to-output planes are shown in Fig. 2(d), (e) for $f=0.6$.

\section{Constant-Frequency, ZV-Quasi-Square-Wave CONVERTERS}

Basic features of CF-ZV-QSW converters are carried over from their frequency-controlled counter-parts--both devices are switching at zero voltage and the switch voltage stress is equal to 1 (equal to the stress in the PWM parent). An example of the CF-ZV-QSW buck topology is shown in Fig. 3(a). An operat- 


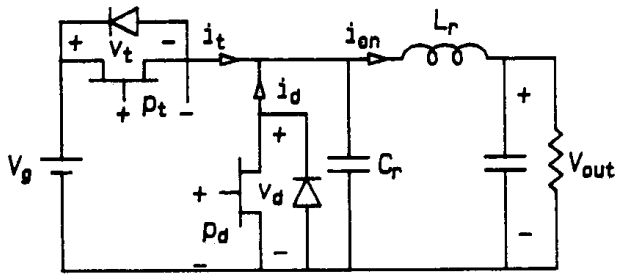

(a)

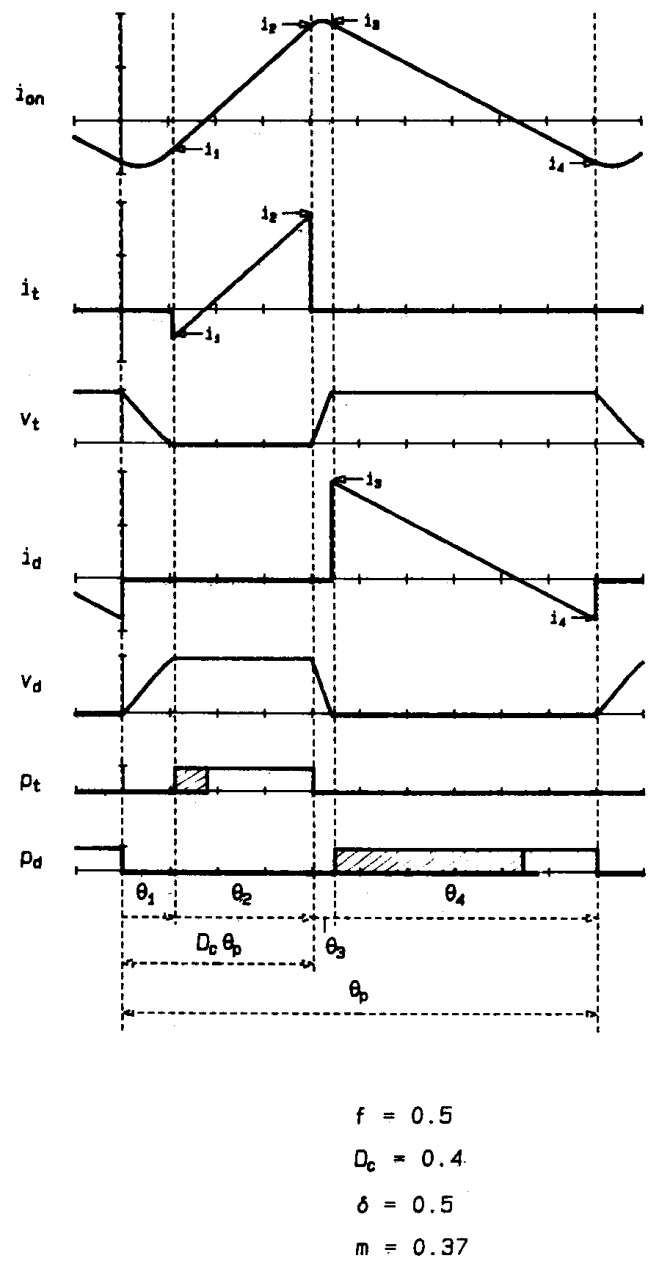

(b)

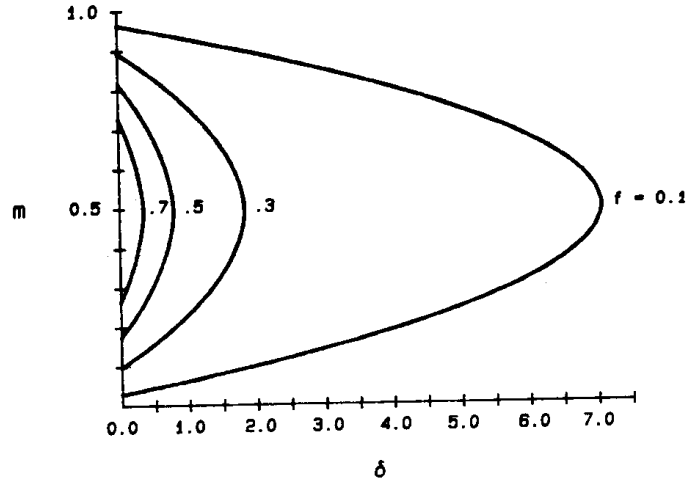

(c)

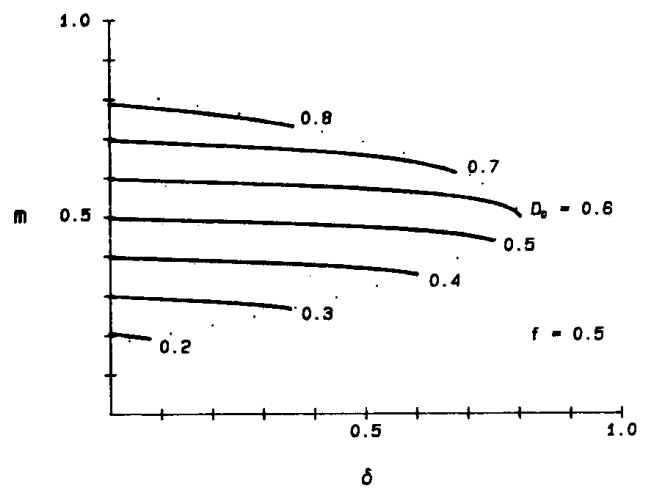

(d)

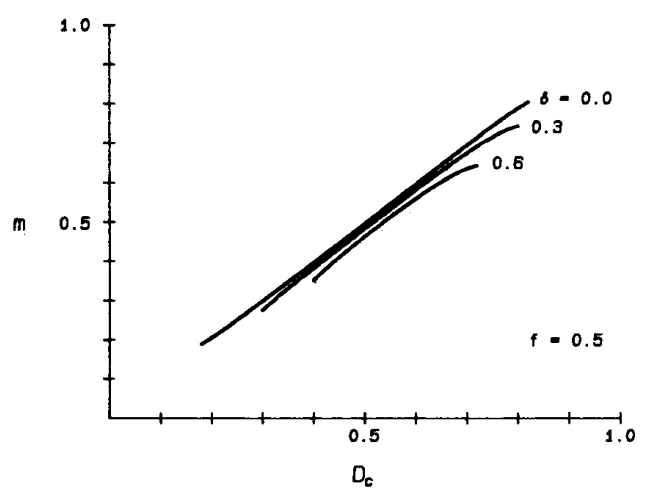

(e)

Fig. 3. (a) CF-ZV-QSW buck converter; (b) typical waveforms in mode $I$; (c) operating region for mode $I$, with normalized frequency $f$ as varying parameter; (d) load-to-output dc characteristics; (e) control-to-output dc characteristics.

ing mode with all zero-voltage transitions (two of which are controllable) is defined by the sequence

$$
I: \cdots \rightarrow 0-0 \rightarrow 1-0 \rightarrow 0-0 \rightarrow 0-1 \rightarrow \cdots,
$$

For a qualitative description of operation in mode $I$ refer to Fig. 3(b). Assume that the converter is initially in the (0-1)-state. When the $\hat{S}$-switch is turned OFF, the (0-0)-state is entered. At the beginning of the (0-0)-state, current $i_{\text {on }}$ is negative and the
$\hat{S}$-switch voltage increases in a quasi-sinusoidal manner. Simultaneously, the $S$-switch voltage decreases until it reaches zero and its antiparallel diode turns on at $\theta=\theta_{1}$. The succeeding (1-0)-state lasts until the $S$-switch is turned ofF at $\theta=\theta_{2}$, initiating the second $(0-0)$-state. Now, the $\hat{S}$-switch voltage descends in a quasi-sinusoidal manner until it drops to zero and its antiparallel diode starts to conduct at $\theta=\theta_{3}$. The converter remains in the (0-1)-state until the $\hat{S}$-switch is turned off again 
and the switching cycle is completed. Note that both turn-off transitions are controllable, while both turn-on transitions are initiated by the circuit waveforms. Hatched areas in the transistor control signals $p_{t}$ and $p_{d}$ denote the intervals when the state of the corresponding transistor can be either ON or OFF, without affecting the operation of the circuit.

Control duty ratio $D_{c}$ is defined as the interval between the $\hat{S}$-switch turn-off and the $S$-switch turn-off relative to the switching period,

$$
D_{c} \equiv \frac{\theta_{1}+\theta_{2}}{\theta_{p}} .
$$

Operating region for mode $I$ is shown in Fig. 3(c) with $f$ as a varying parameter. The region includes a part of the zero-load axis and it is symmetrical around $m=0.5$. This is a significant improvement over the frequency-controlled ZV-QSW converters for which the operating region is limited to $m \geq 0.5$ [4]. The operating region can be expanded in both $\delta$ and $m$ directions by selecting a lower value for the normalized frequency $f$. In addition, the switch current stresses (relative to the maximum output current) are lower for lower $f$ but relative lengths of the (0-0)-states (transition states) also become shorter. As the lengths of the transition states become comparable to switching times of the utilized devices, the reduction in switching losses achieved by the zero-voltage switching is adversely affected. In the limit, if $f \rightarrow 0$, mode $I$ becomes identical to the continuous conduction mode of the PWM parent-transition intervals shrink to zero and the lossless zero-voltage switching is lost. Thus, the choice of $f$ is determined by the required range of accessible equivalent duty ratios and by the trade-off between current stresses and the lengths of the transition states.

DC characteristics of a CF-ZV-QSW converter are shown in Fig. 3(d), (e) for $f=0.5$. Except for the limitation in the operating region, the characteristics are similar to the characteristics of the PWM parent. Inside the operating region in the load-to-output plane, the dc characteristics are almost horizontal lines, i.e., the output voltage is weakly dependent on the output current.

\section{Constant-Frequency, ZV-Multi-Resonant CONVERTERS}

In a CF-ZV-MR converter, both switches can be turned oN and $\mathrm{OFF}$ at zero voltage. In addition, all major parasitic elements-junction capacitances of the switches and the parasitic inductance distributed in the loop with the switches (including the transformer leakage inductance, if an isolation transformer is used)-can be incorporated in the resonant network. Both features are inherited from the conventional, frequency-controlled ZV-MR converters. An example of the CF-ZV-MR buck converter is shown in Fig. 4(a). Parameter $x$ is the ratio of the capacitances of the two resonant capacitors-the capacitor across the $\hat{S}$-switch and the capacitor across the $S$-switch. There are four operating modes of interest:

$$
\begin{aligned}
& I: \cdots \rightarrow 1-1 \rightarrow 1-0 \rightarrow 0-0 \rightarrow 0-1 \rightarrow \cdots, \\
& I I: \cdots \rightarrow 1-1 \rightarrow 1-0 \rightarrow 0-0 \rightarrow 1-0 \rightarrow \cdots, \\
& I I I: \cdots \rightarrow 1-1 \rightarrow 0-1 \rightarrow 0-0 \rightarrow 0-1 \rightarrow \cdots, \\
& I V: \cdots \rightarrow 1-1 \rightarrow 0-1 \rightarrow 1-1 \rightarrow 1-0 \rightarrow \cdots .
\end{aligned}
$$

Let us examine the operation in mode III for which pertinent waveforms are shown in Fig. 4(b). Assume that the CF-ZV-
MR converter is initially in the (1-1)-state. The (0-1)-state is entered when the $S$-switch is turned OFF at zero voltage, at $\theta=$ $\theta_{1}$. Capacitor $C_{r}$ and inductor $L_{r}$ are allowed to resonate. The $S$-switch voltage $\left(v_{t}\right)$ starts from zero and increases in a quasisinusoidal manner. The converter remains in the (0-1)-state until the $\hat{S}$-switch is turned off at zero voltage, at $\theta=\theta_{2}$, so that the next $(0-0)$-state is entered. The $(0-0)$-state is the multiresonant state in which all three resonant elements $\left(C_{r}, x C_{r}\right.$ and $L_{r}$ ) are allowed to resonate. With the initial conditions from the $(0-1)$-state; $v_{t}>0, v_{d}=0$, the next state is necessarily the (0-1)-state, i.e., the multi-resonant state terminates when the $\hat{S}$-switch voltage drops to zero, and the antiparallel diode in the $\hat{S}$-switch starts to conduct. In the (0-1)-state, the $\hat{S}$-switch voltage $\left(v_{d}\right)$ is clamped to zero, while $C_{r}$ and $L_{r}$ continue to resonate so that the $s$-switch voltage $\left(v_{t}\right)$ decreases toward zero. The switching cycle is completed at $\theta=\theta_{4}$ when voltage $v_{t}$ reaches zero and the antiparallel diode in the $S$-switch starts to conduct. After the (1-1)-state is entered, the transistor in the $S$-switch has to be turned on before the $S$-switch current $\left(i_{t}\right)$ becomes positive.

During the intervals when the antiparallel diode of a switch is oN, the state of the corresponding transistor is irrelevant for the operation of the circuit, as indicated by the hatched areas in the transistor control signals $p_{t}$ and $p_{d}$. A qualitative description of the operation in the other three modes $(I, I I$, and $I V)$ can be deduced in a similar manner as for mode III.

For any of the four operating modes, the part of the switching cycle between the two controllable transitions (the $\hat{S}$-switch turn-off and the $S$-switch turn-off) defines the control variable $D_{c}$,

$$
D_{c}= \begin{cases}\theta_{2} / \theta_{p}, & \text { modes } I \text { and } I I, \\ -\theta_{2} / \theta_{p}, & \text { mode } I I I \\ -\left(\theta_{2}+\theta_{3}\right) / \theta_{p}, & \text { mode } I V .\end{cases}
$$

As far as external properties of a CF-ZV-MR converter are concerned, it is convenient to treat operation in modes $I, I I$, and $I I I$ as if the converter operated in a single mode. The combined operating region is shown in Fig. 4(b) with normalized frequency $f$ as a varying parameter and with the ratio of resonant capacitors $(x)$ set to 1 . From zero load up to some maximum load $\delta_{\max }(f)$, all equivalent duty ratios are accessible, provided that $f \leq 1$. The maximum load is larger and the current stress is lower for lower $f$. However, for lower $f$, the peak switch voltage stresses are higher, as indicated in Table II. For $x=1$, the peak stresses for $S$ and $\hat{S}$ are identical. A sensible guideline for practical designs is to choose $f$ in the range $0.9<f<1$ so that the operating region spans all possible equivalent duty ratios while the voltage stress is minimized. For $f$ in this range, the computed voltage and current stresses compare favorably to the stresses reported in [5] for conventional frequency-controlled ZV-MR converters.

Combined dc characteristics for modes $I, I I$, and $I I I$ are shown in Fig. 4(d), (e) for $f=0.9$ and $x=1$.

Mode $I V$ is of less practical interest because the operating region is more restricted while switch stresses are higher [12].

\section{An Experimental CF-ZV-MR Buck Converter}

CF-ZV-MR converters are particularly interesting for highfrequency applications - the topology is insensitive to all major circuit parasitics; all switching transitions are at zero voltage; 


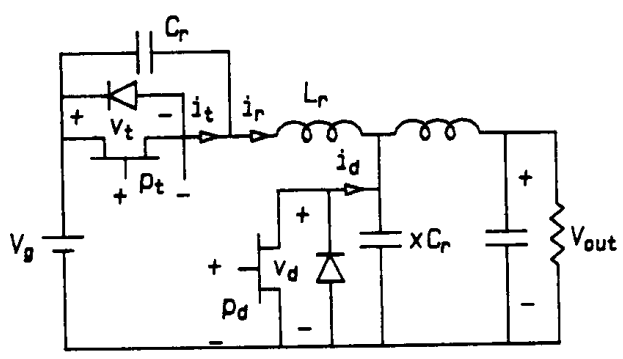

(a)

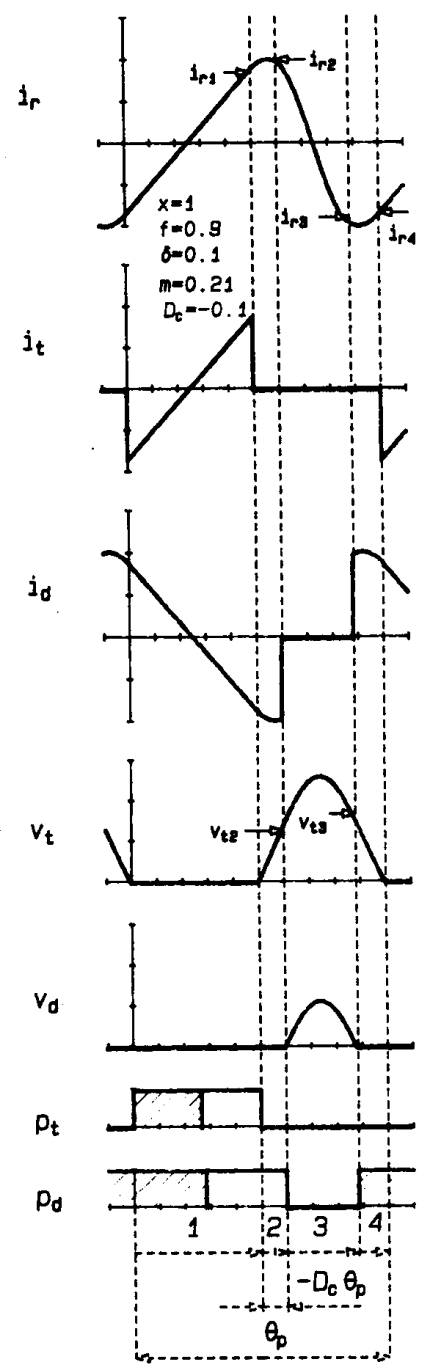

(b)

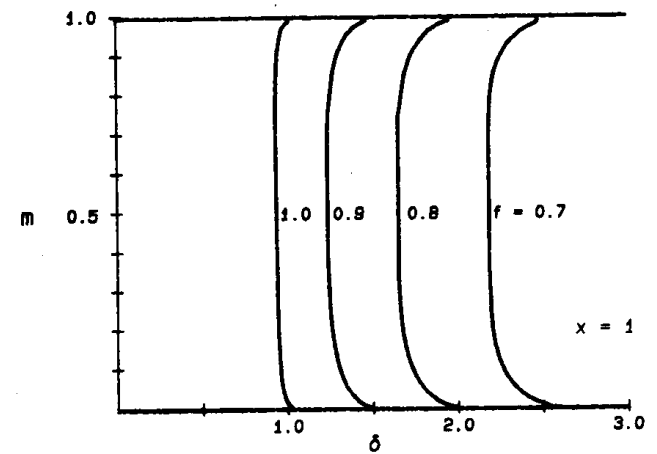

(c)

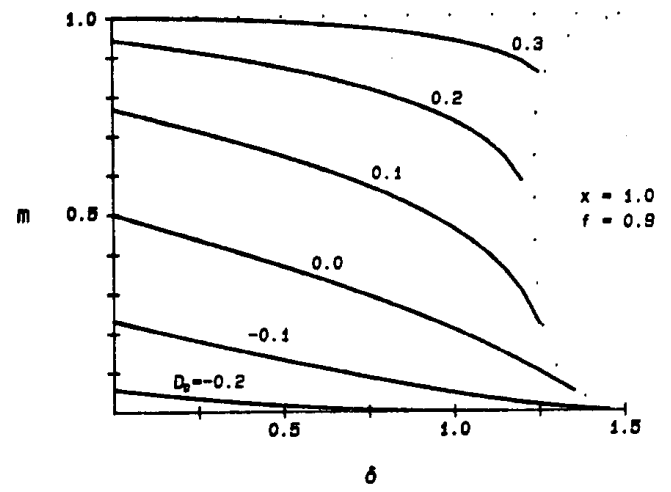

(d)

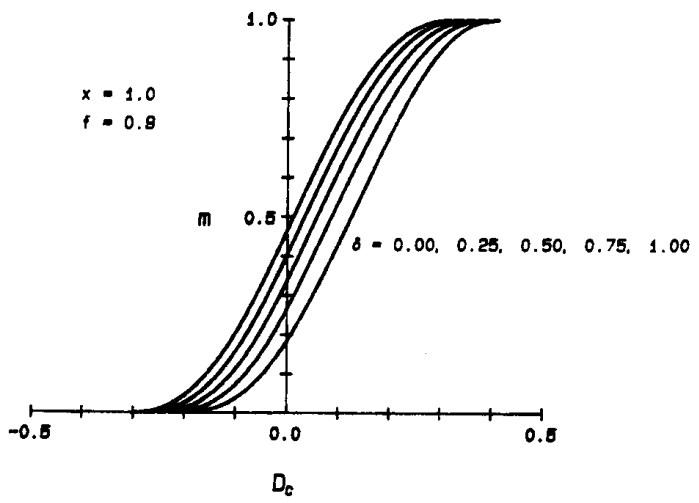

(e)

Fig. 4. (a) CF-ZV-MR buck converter; (b) typical waveforms in mode $I I$; (c) combined operating regions for modes $I, I I$, and III, with normalized frequency $f$ as varying parameter; (d) load-to-output dc characteristics; (e) control-to-output dc characteristics.

full load range $0<\delta<\delta_{\max }$ and full conversion range $0 \leq m$ $\leq 1$ are attainable; finally, voltage and current stresses are moderate. This is why a CF-ZV-MR buck converter is selected for experimental evaluation.
The circuit diagram of the experimental CF-ZV-MR buck converter operating at $f_{s}=2 \mathrm{MHz}$ is shown in Fig. 5. The converter is designed for $V_{g}=20 \mathrm{~V}, V_{\text {out }}=10 \mathrm{~V}$ and a maximum load current of $2.5 \mathrm{~A}$. After $x=1$ and the switching fre- 


\begin{tabular}{cccc}
\multicolumn{4}{c}{ TABLE II } \\
\hline$f$ & $V_{\text {stress }}$ & $\delta_{\text {max }}$ & $I_{\text {stress }}$ \\
\hline 0.6 & 4.5 & 2.9 & 1.6 \\
0.7 & 3.8 & 2.2 & 1.7 \\
0.8 & 3.2 & 1.7 & 1.9 \\
0.9 & 2.7 & 1.3 & 2.1 \\
1.0 & 2.3 & 1.0 & 2.4 \\
\hline
\end{tabular}

Switch voltage stress ( $\left.V_{\text {stress }}\right)$, maximum normalized load $\left(\phi_{\max }\right)$, and switch current stress $\left(I_{\text {stress }}\right)$ as functions of the normalized frequency $f$ for $x=1$. The stresses are computed relative to the stresses in the PWM parent.

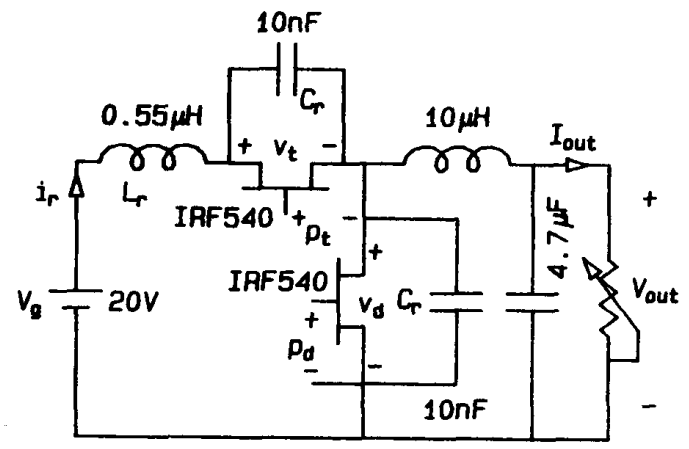

Fig. 5. Experimental CF-ZV-MR buck converter.

quency are selected, the design of a CF-ZV-MR converter reduces to the choice of values for parameters $f$ and $R_{o}$. Normalized frequency $f=0.95$ is selected to insure that the operating region spans the full range of conversion ratios, while the switching devices are subject to voltage stresses close to the minimum values. Parameter $R_{o}$ is chosen so that the maximum load current is inside the available operating region:

$$
\delta_{\max }(f) \frac{V_{g}}{R_{o}} \geq\left(I_{\text {out }}\right)_{\max } \Rightarrow R_{o} \leq 8 \Omega .
$$

In the design for the maximum output current, some safety margin is required because all results in Table II of Section V were derived assuming that the converter is lossless. Thus, we select $R_{o}=7.5 \Omega$. Once $f$ and $R_{o}$ are determined, the values of the resonant elements are given by

$$
\begin{aligned}
& L_{r}=\frac{1}{2 \pi} \frac{f}{f_{s}} R_{o}=0.56 \mu \mathrm{H}, \\
& C_{r}=\frac{1}{2 \pi} \frac{f}{f_{s}} \frac{1}{R_{o}}=10.1 n \mathrm{~F},
\end{aligned}
$$

and the design is completed.

In the first experiment, the converter is operated at nominal output voltage from no load to maximum load. Duty ratio of the $S$-switch $\left(D_{t}\right)$ is kept constant $\left(D_{t} \approx 0.4\right)$, while duty ratio of the $\hat{S}$-switch $\left(D_{d}\right)$ is adjusted so that $V_{\text {out }}=10 \mathrm{~V}$ is maintained. Since the transistor control signals are synchronized on their leading edges, the control duty ratio $\left(D_{c}\right)$ is equal to the difference between the two duty ratios,

$$
D_{c}=D_{d}-D_{r} \text {. }
$$

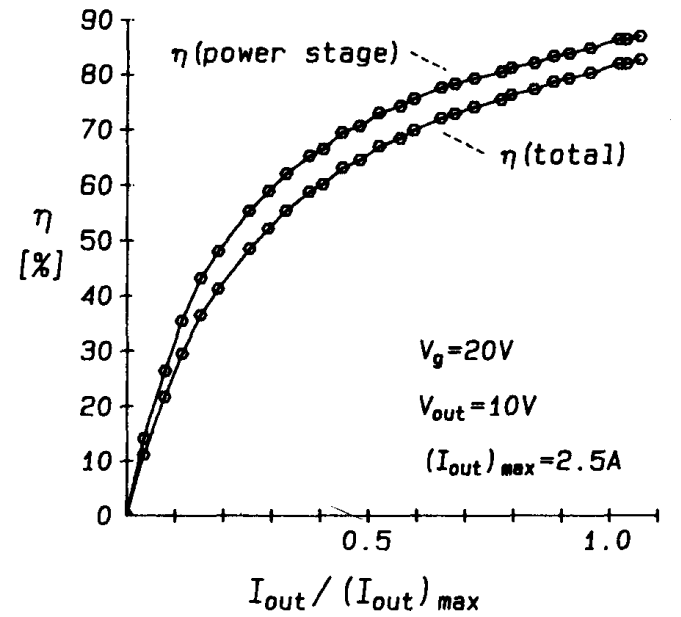

Fig. 6. Efficiency of power stage alone and total efficiency of experimental CF-ZV-MR buck converter as functions of load current.

It is interesting that the total power loss in the circuit is almost constant throughout the load range. Thus, the maximum efficiency is obtained at maximum load $-87 \%$ for the power stage alone (excluding the gate-drive loss $P_{g}$ ), or $83 \%$ for the complete converter. For $f_{s}=2 \mathrm{MHz}, V_{g s}=10 \mathrm{~V}$ and $Q_{g s}$ (IRF540) $=40 \mathrm{nC}$, the total gate-drive loss,

$$
P_{g}=2 f_{s} V_{g s} Q_{g s}=1.6 \mathrm{~W}
$$

contributes significantly to the total power loss. A quasi-resonant gate drive [11] could be used to reduce the gate drive loss by half. Measured efficiency as a function of the load current is shown in Fig. 6.

Throughout the load range, the maximum measured voltage stress on both switching devices is less than $50 \mathrm{~V}$, which is in agreement with the theoretical prediction.

In the second experiment, duty ratios of both switches are kept constant at the values that result in the maximum output current at the nominal output voltage. Then, the output load is changed from open circuit to short circuit. Measured output voltage and output power as functions of the load current are shown in Fig. 7.

A remarkable feature of the CF-ZV-MR converter is that it behaves as a constant current source after the maximum output current is exceeded. Thus, the output short-circuit current (2.67 A) is only slightly higher than the maximum nominal load current. If the maximum control duty ratio is limited, the converter is immune to both no-load and short-circuit conditions without any additional protection. The output power collapses abruptly after the load resistor is decreased beyond the value that corresponds to the maximum output power. The power loss in the converter is approximately constant throughout the load range. Thus, when the output is shorted, the power taken from the input source is approximately the same as the power taken under the no-load condition $\left(P_{\text {loss }} \approx 4 \mathrm{~W}\right)$.

Waveforms recorded for three operating points-no load (A), maximum output current at $V_{\text {out }}=10 \mathrm{~V}$ (B), and short circuit (C)-are shown in Fig. 8. Note that the converter operates in mode $I I$ at point $\mathrm{A}$, in mode $I$ at point $\mathrm{B}$ and in mode $I I I$ at point C. 


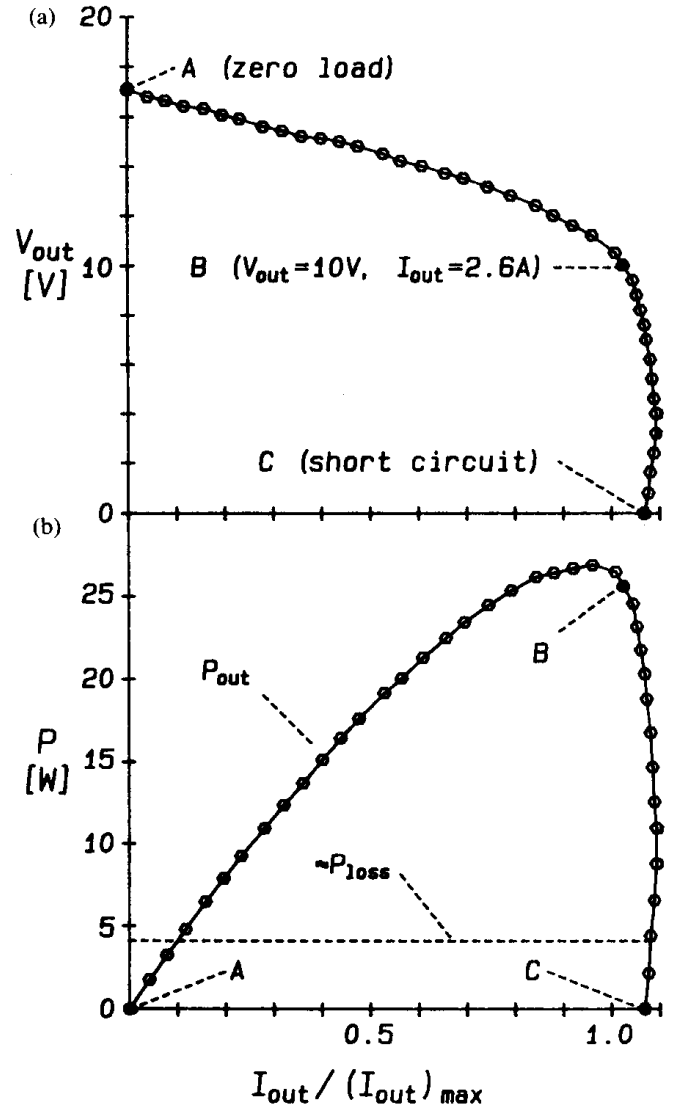

Fig. 7. Output voltage (a) and output power (b) as functions of load current for constant control duty ratio, $D_{c} \approx 0.16$

\section{CONClusion}

A constant-frequency quasi-resonant (CF-QR) converter is constructed by replacing the diode switch with a controllable rectifier in any quasi-resonant topology. The controllable rectifier provides an additional independent control needed for constant-frequency operation throughout the load range. The suggested modification can be applied to all classes of quasiresonant converters and it leads to a number of novel operating modes of practical interest.

Compared to QR converters with conventional switch realization, CF-QR converters exhibit the same type of switching transitions and similar switch voltage and current stresses. Advantages of CF-QR converters are not restricted to the constantfrequency control. In all classes, operation at zero load is possible, so that the available load range is unlimited. Furthermore, the range of attainable conversion ratios is significantly extended in the classes of zero-voltage quasi-square-wave (CF$\mathrm{ZV}-\mathrm{QSW}$ ) and zero-voltage multi-resonant (CF-ZR-MR) topologies.

As a practical design example, a $25 \mathrm{~W}$ CF-ZV-MR buck converter is constructed and evaluated. The converter operates at 2 $\mathrm{MHz}$ from zero load to full load, with a full-load efficiency of $83 \%$. Simple duty ratio control is used to maintain the output voltage constant for all loads. The circuit is inherently immune to the short-circuit condition at the output-the short-circuit current is only slightly higher than the nominal full-load cur-

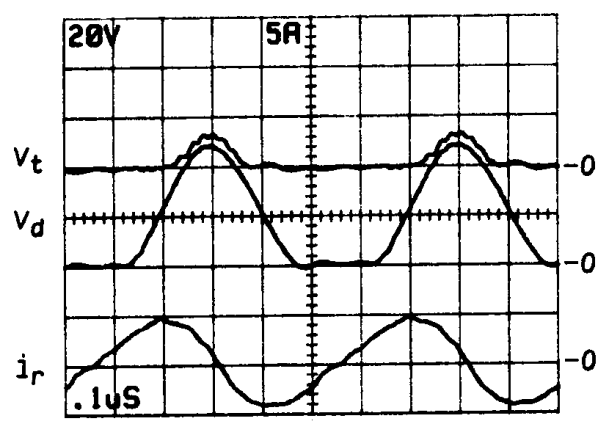

(a)

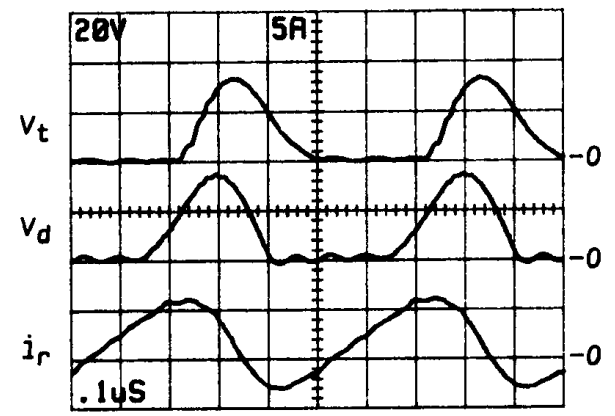

(b)

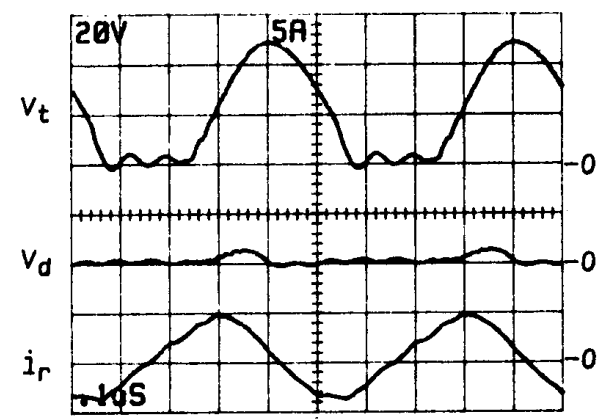

(c)

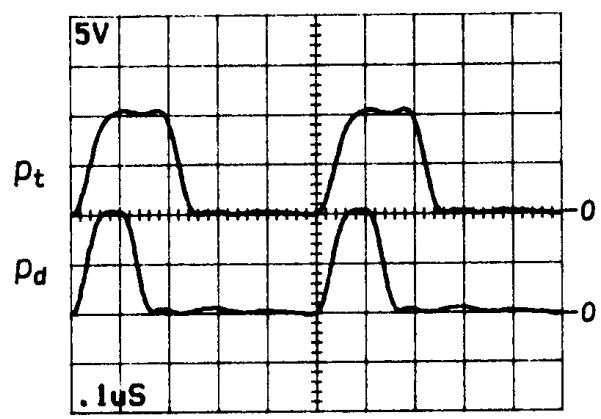

(d)

Fig. 8. Waveforms recorded in experimental CF-ZV-MR converter at (a) no load (operating point $\mathrm{A}$ ); (b) full nominal load (operating point B); (c) short circuit (operating point $C$ ). (d) Transistor drive waveforms.

rent, and the power loss in the circuit is approximately the same as under the zero-load condition.

Disadvantages of CF-QR converters are the increased gatedrive losses and increased complexity of the power stage and 
the control circuitry. One should note, however, that if the use of controllable rectifiers by the reduction in conduction losses (as suggested by the concept of synchronous rectification) all other benefits of CF-QR converters come virtually without penalty. Thus, with further developments of ultra-low-on-resistance MOS transistors, CF-QR topologies become particularly suitable for very-high-density, low-output-voltage power supplies.

\section{REFERENCES}

[1] K.-H. Liu and F. C. Lee, "Resonant switches-a unified approach to improve performance of switching converters," in Proc. IEEE INTELEC, 1984, pp. 344-351.

[2] K.-H. Liu and F. C. Lee, "Zero-voltage-switching technique in $\mathrm{dc} / \mathrm{dc}$ converters," in IEEE/PESC Conf. Rec., 1986, pp. 58-70.

[3] K. D. T. Ngo, "Generalization of resonant switches and quasiresonant dc-dc converters," in IEEE/PESC Conf. Rec., 1987, pp. 395-403.

[4] V. Vorperian, "Quasi-square-wave converters: Topologies and analysis," Proc. VPEC, 1987.

[5] W. A. Tabisz and F. C. Lee, ' Zero-voltage-switching multi-resonant technique-A novel approach to improve performance of high-frequency quasi-resonant converters," in IEEE/PESC Conf. Rec., 1988.

[6] K. Harada, W. J. Gu, and K. Murata, "Controlled resonant converters with switching frequency fixed," in IEEE/PESC Conf. Rec., 1987, pp. 431-438,
[7] S. G. Trabert and R. W. Erickson, "Steady state analysis of the duty cycle controlled series resonant converter," in IEEE/PESC Conf. Rec., 1987, pp. 545-556.

[8] O. D. Patterson and D. M. Divan, "Pseudo-resonant Full Bridge $\mathrm{dc} / \mathrm{dc}$ converter," in IEEE/PESC Conf. Rec., 1987, pp. 424-430.

[9] C. P. Henze, H. C. Martin, and D. W. Parsley, "Zero-voltage switching in high frequency power converters using pulse width modulation," Proc. IEEE/APEC, 1988, pp. 33-40.

[10] S. Freeland and R. D. Middlebrook, "A unified analysis of converters with resonant switches,"' in IEEE/PESC Conf. Rec., 1987, pp. $20-30$.

[11] W. A. Tabisz, P. Gradzki, and F. C. Lee, "Zero-voltageswitched quasi-resonant buck and flyback converters-experimental results at $10 \mathrm{MHz}, "$ in IEEE/PESC Conf. Rec., 1987, pp. 404-413.

[12] D. Maksimović, "Synthesis of PWM and quasi-resonant de-todc power converters," Ph.D. dissertation, California Institute of Technology, Pasadena, 1989.

[13] D. Maksimović and S. Cuk, "A general approach to synthesis and analysis of quasi-resonant converters, " in IEEE/PESC Conf. Rec., 1989, pp. 713-727.

Dragan Maksimović (M'89). For a photograph and biography please turn to page 140 of this issue.

Slobodan Ćuk (M'74). For a photograph and biography please turn to page 140 of this issue. 